\title{
GDC-0326 Enhances the Effects of 5-Fu in Colorectal Cancer Cells by Inducing Necroptotic Death
}

\author{
Zizhen Zhang ${ }^{1-3, *}$ \\ Fangyu Ju ${ }^{1-3, *}$ \\ Fei Chen ${ }^{1-3}$ \\ Haoyue $\mathrm{Wu}^{4}$ \\ Jingyu Chen ${ }^{1-3}$ \\ Jing Zhong ${ }^{1-3}$ \\ Liming Shao ${ }^{1-3}$ \\ Sheng Zheng ${ }^{1-3}$ \\ Liangjing Wang ${ }^{1-3}$ \\ Meng Xue (iD ${ }^{1-3}$ \\ 'Department of Gastroenterology, The \\ Second Affiliated Hospital of Zhejiang \\ University School of Medicine, Hangzhou, \\ 310020, People's Republic of China; \\ ${ }^{2}$ Institution of Gastroenterology, Zhejiang \\ University, Hangzhou, 310000, People's \\ Republic of China; ${ }^{3}$ Zhejiang University \\ Cancer Center, Hangzhou, 310000, \\ People's Republic of China; ${ }^{4}$ Institute of \\ Genetics and Department of Genetics, \\ Zhejiang University School of Medicine, \\ Hangzhou, 310012, People's Republic of \\ China
}

*These authors contributed equally to this work
This article was published in the following Dove Press journal: OncoTargets and Therapy

\begin{abstract}
Aim: Chemoresistance to 5-fluorouracil $(5-\mathrm{Fu})$ is common in colorectal cancer (CRC). Programmed necrosis (necroptosis) is an alternative form of programmed cell death regulated by receptor-interacting protein kinase (RIPK) 1 and 3, assumed as a novel target of cancer therapy. In this study, we aimed to explore whether a novel small molecular agent GDC-0326 could facilitate the effect of 5-Fu through necroptosis.
\end{abstract}

Main Methods: Cell Counting Kit-8 (CCK-8) assay and colony formation were performed to confirm the function of GDC-0326 in CRC cells. Western blot and immunofluorescence were conducted to measure the altered expressions of RIPK1/RIPK3 induced by GDC-0326. Subcutaneous tumor models were used to evaluate the chemotherapeutic effects and concomitant side effects of GDC-0326 in vivo.

Key Findings: We found that GDC-0326 effectively suppressed the growth of CRC cells in a dose-dependent manner. The induction of necroptosis by GDC-0326 was correlated with the modulation of RIPK1 and RIPK3. Necrostatin-1 and GSK-872, inhibitors of RIPK1 and RIPK3, respectively, could rescue the cell death induced by GDC-0326. In addition, in vitro and in vivo studies showed that 5-Fu plus GDC-0326 evinced a better antitumor efficacy by suppressing tumor growth and increasing tumor necrosis with no increased toxicity.

Significance: This study demonstrates that GDC-0326 plus 5-Fu has augmented antitumor efficacy and acceptable safety, which might be a promising therapeutic strategy for CRC patients in the future.

Keywords: GDC-0326, 5-fluorouracil, colorectal cancer, combination therapy

\section{Introduction}

Despite improvements in the diagnosis and treatment of colorectal cancer (CRC), it remains the fourth most common cancer and the second leading cause of cancerrelated mortalities globally, especially in Western countries. ${ }^{1-3}$ 5-Fluorouracil $(5-\mathrm{Fu})$ is a classic and widely used first-line chemotherapy drug for CRC. However, a fairly large proportion of CRC patients develop resistance to 5-Fu, and this impairs their life quality and prognosis. Thus, it is important to uncover effective methods to reduce resistance to $5-\mathrm{Fu}$ and improve the therapeutic sensitivity of CRC patients treated with 5-Fu.

Some common mechanisms of drug resistance include activation of efflux transporters, dysregulation of metabolism, enhancement of DNA repair capacity, alteration of vital signal pathways essential for proliferation, and resistance to drug-induced apoptosis. ${ }^{4,5}$ Most of the previously reported strategies focus on
Correspondence: Meng Xue; Liangjing Wang Email xuemeng@zju.edu.cn; wangljzju@zju.edu.cn
OncoTargets and Therapy 2021:14 2519-2530

2519 
restoring the sensitivity of chemotherapeutic agents. For instance, several small molecular compounds, including S1PR2 inhibitors and andrographolide, can reverse 5-Fu resistance by increasing the apoptosis of tumor cells. ${ }^{6-8}$ Further, in esophageal squamous cell carcinoma, the efficacy of $5-\mathrm{Fu}$ was enhanced with the addition of LY294002, an inhibitor of autophagy. ${ }^{9}$ Another study showed that knockdown of the lncRNA NEAT1 attenuated autophagy and elevated 5-Fu sensitivity in CRC. ${ }^{10}$ In addition, a specific pro-inflammatory form of regulated cell death, called pyroptosis, was found to enhance antitumor immunity via activation of Gasdermin E. ${ }^{11,12}$ Thus, targeting the cell death pathways of tumor cells seems to be a promising strategy for improving the therapeutic efficacy to 5 -Fu.

Inducing apoptosis is the main cytotoxic mechanism of 5-Fu, but other forms of programmed cell death have gained increasing attention in recent years. ${ }^{13-15}$ For example, necroptosis is a form of necrotic cell death that is distinguished from apoptosis by a cascade of signaling pathways that do not involve the activation of caspase proteases. Studies have demonstrated that necroptosis is a new therapeutic target in cancer, including breast cancer, glioma and lung cancer. ${ }^{16,17}$ With regard to the mechanisms involved, receptor interacting serine/threonine kinase 1 (RIPK1) and receptor interacting serine/threonine kinase 3 (RIPK3) play critical roles in this type of cell death. ${ }^{18}$ Thus, induction of necroptosis via RIPK1 and RIPK3 might be an effective way to overcome apoptosis resistance in cancer cells. ${ }^{19}$

$\mathrm{PI} 3 \mathrm{~K} \alpha$, which is a member of the PI3 kinase family, has been found to play a role in cancer cell proliferation and cancer progression. ${ }^{20-22}$ One study has shown that the PI3K/AKT pathway is associated with necroptosis. ${ }^{23}$ Moreover, selective inactivation of $\mathrm{PI} 3 \mathrm{~K} \alpha$ has been found to inhibit the proliferation and migration of pancreatic cancer cells. ${ }^{24}$ Therefore, inhibiting the PI3K/AKT signaling pathway may lead to an increase in necroptotic cell death. Additionally, the resulting increase in necroptosis may involve activation of RIPK1 and RIPK3. Based on these findings, it would be interesting to explore whether inhibition of PI3K might improve therapeutic efficacy to 5 -Fu by causing an increase in necroptosis in CRC via the activation of RIPK1/3.

GDC-0326 is a PI3K $\alpha$-specific inhibitor that exhibits better selectivity for PI3K $\alpha$ than for other PI3 kinases. ${ }^{25}$ Therefore, in the present study, we have examined whether GDC-0326 can be used as a potential therapeutic adjuvant along with 5-Fu for the treatment of CRC via induction of necroptosis, and we have also investigated whether RIPK1/3 might be involved in any observed necroptotic effects of GDC-0326.

\section{Materials and Methods Cell Lines and Cell Culture}

CRC cell lines (LoVo and HT-29) were purchased from American Type Culture Collection (ATCC, MA, USA). HT-29 cells were maintained in McCoy's 5A medium (Genom, Hangzhou, China) supplemented with 10\% fetal bovine serum (FBS; Sijiqing, Hangzhou, China), and LoVo cells were cultured in $\mathrm{F}-12 \mathrm{~K}$ medium (Genom) containing 10\% FBS. All the CRC cell lines were cultured in a humidified atmosphere containing $5 \% \mathrm{CO}_{2}$ at $37^{\circ} \mathrm{C}$.

\section{Western Blotting Analysis}

For Western blotting analysis, total proteins were extracted from the CRC cell lines. Protein was loaded on $10 \%$ sodium dodecyl sulfate polyacrylamide gels, transferred to polyvinylidene fluoride (PVDF) membranes, blocked with $5 \%$ fat-free milk for $1 \mathrm{~h}$, and incubated with primary antibodies at $4{ }^{\circ} \mathrm{C}$ overnight. The following primary antibodies were used: RIPK1 (diluted 1:1000, \#3493; CST, Danvers, USA), RIPK3 (diluted 1:100, sc-374,639; Santa Cruz Biotechnology, Santa Cruz, USA), AKT (diluted 1:1000, \#9272; CST, Danvers, USA), P-AKT (ser473) (diluted 1:1000, AP0637; ABclonal Biotechnology, Wuhan, China) PI3K (diluted 1:1000, \#4255; CST) and $\beta$ actin (as the loading control) (diluted 1:1000, \#3700; CST). The PVDF membranes were then incubated with secondary antibodies labeled with horse radish peroxide at room temperature. The blots were detected with an enhanced chemiluminescence kit (Fdbio science, Hangzhou China).

\section{Cell Viability Assay}

Cell viability was measured using Cell Counting Kit-8 (CCK-8; Dojin Laboratories, Kumamoto, Japan) according to the manufacturer's instructions. Briefly, LoVo and HT29 cells were seeded at a density of $5 \times 10^{3}$ cells/well at the bottom of 96-well plates. Nec-1 (an inhibitor of RIPK1), GSK-872 (an inhibitor of RIPK3), or DMSO (control), in combination with various concentrations $(0.4-10 \mu \mathrm{M})$ of GDC-0326 were added to $100 \mu \mathrm{L}$ of complete media. Cells were then incubated with $10 \mu \mathrm{L}$ of CCK-8 solution for $2 \mathrm{~h}$ at $37^{\circ} \mathrm{C}$. Finally, the absorbance 
at $450 \mathrm{~nm}$ was measured using a microplate reader (Model 680; Bio-Rad, Hercules, CA, USA). The $\mathrm{IC}_{50}$ value of GDC-0326 was determined based on the cell viability.

\section{Analysis of the Synergistic Effect of GDC-0326 and 5-Fu}

Based on the $\mathrm{IC}_{50}$ determined for GDC-0326, different concentrations of GDC-0326 and 5-Fu were mixed at the same ratio to determine the synergetic dose-effect relationship. The combination index (CI) was assessed with the Chou-Talalay combination index (CI) theorem, ${ }^{26}$ and based on it, the effect was quantitatively evaluated as follows, using the CompuSyn software: $\mathrm{CI}<1$, synergistic effect; $\mathrm{CI}=1$, additive effect; $\mathrm{CI}>1$, antagonistic effect.

\section{Colony Formation Assay}

LoVo and HT-29 cells were plated at a final density of $300-500$ cells/well in a six-well plate in complete growth media and cultivated for $24 \mathrm{~h}$. Then, different doses of GDC-0326 were added to each well. The growth medium was changed every 3 days until visible colonies were formed. Colonies were washed twice with phosphatebuffered saline (PBS), fixed with ethanol, and stained with $0.05 \%$ crystal violet in $20 \%$ ethanol. The number of colonies that contained more than 50 cells was counted.

\section{Immunofluorescence Assay}

After treatment with different concentrations of GDC0326, cells growing on coverslips were washed with PBS, fixed with 4\% paraformaldehyde for $10 \mathrm{~min}$, permeabilized with $0.1 \%$ Triton $\mathrm{X}-100$, and blocked for $1 \mathrm{~h}$ in PBS containing $0.1 \%$ Tween 20 (PBST) and 5\% bovine serum albumin. Subsequently, cells were incubated overnight with primary antibodies (RIPK1 diluted to $1: 100$ or RIPK3 diluted to $1: 50$ ) at $4{ }^{\circ} \mathrm{C}$ overnight and then washed with PBST. Next, samples were incubated with AF488conjugated rabbit secondary antibody (1:500; Beyotime, Shanghai, China) and Cy3-labeled secondary antibody (1:1000, Beyotime) for $1 \mathrm{~h}$ at room temperature, and then counterstained with 4',6-diamidino-2-phenylindole (DAPI) to visualize the nucleus. Images were observed and analyzed using an Olympus FV1000 confocal laser scanning microscope.

\section{In vivo Mouse Models}

All animal experiments were performed under the guidelines of the Institutional Animal Care and Use
Committee of the Second Affiliated Hospital of Zhejiang University School of Medicine. All procedures were approved by the Ethics Committee of the Second Affiliated Hospital of Zhejiang University School of Medicine. A xenograft model was generated by subcutaneous injection of colon cancer cells of the LoVo cell line $\left(2 \times 10^{6}\right.$ cells suspended in $200 \mu \mathrm{L}$ PBS $)$ into 6 -week-old female $\mathrm{BALB} / \mathrm{c}$ nude mice. When the tumors became palpable $\left(200 \mathrm{~mm}^{3}\right)$, the mice were randomly assigned to the following treatment groups ( $\mathrm{n}=5$ in each group): (I) vehicle, (II) $5-\mathrm{Fu}(10 \mathrm{mg} /$ $\mathrm{kg})$, (III) GDC-0326 (6.25 $\mathrm{mg} / \mathrm{kg})$, and (IV) a combination of $5-\mathrm{Fu}(10 \mathrm{mg} / \mathrm{kg})+$ GDC-0326 (6.25 $\mathrm{mg} / \mathrm{kg})$. 5-Fu was intraperitoneally injected, while GDC-0326 was administered orally. All the drugs were administered once every 2 days over a 12day period. Tumor size was assessed every 2 days with digital calipers. Tumor volume was calculated with the following formula: $\mathrm{V}\left(\mathrm{mm}^{3}\right)=\left(\right.$ width $^{2} \times$ length $) / 2$. The curve of tumor growth was drawn based on tumor volume and corresponding time (days) after treatment. Body weight was simultaneously measured. Tumor weights were measured using an electronic scale after the mice were sacrificed. Tumors were harvested for TdT-mediated dUTP nick end labeling (TUNEL) and hematoxylin and eosin (H\&E) staining. Half of each tumor was frozen in liquid nitrogen for protein isolation later.

\section{Complete Blood Count and Serum Biochemistry Analysis}

At the end of the experiment, whole blood was collected from the eyeball. We then analyzed the total number of white blood cells (WBCs), red blood cells (RBCs), hemoglobin, and platelets (PLT), and measured the serologic parameters associated with kidney and liver function, including blood urea nitrogen (BUN), creatinine $(\mathrm{Cr})$, aspartate aminotransferase (AST), alanine aminotransferase (ALT), and total protein (TP).

\section{Validation and Prognostic Analysis}

Data on the expression of RIPK1/3 and clinical features of CRC were downloaded from The Cancer Genome Atlas (TCGA) database (https://portal.gdc.cancer.gov/). KaplanMeier survival analysis was used to estimate and compare survival in patients with high and low expression of RIPK1 and RIPK3. 


\section{Statistical Analysis}

Experimental results were expressed as mean \pm standard deviation (SD). All statistical analyses were performed using SPSS 22.0 or GRAPHPAD PRISM, version 7.0. Statistical significance was calculated using a two-tailed Student's $t$-test or analysis of variance (ANOVA). A P value of less than 0.05 was considered statistically significant.

\section{Results}

\section{Inhibition of CRC Cell Proliferation by GDC-0326 via Upregulation of RIPKI/3}

When cells of the two human CRC cell lines LoVo and HT-29 were treated with various concentrations of GDC0326 for $48 \mathrm{~h}$ and cell viability was assessed, dosedependent growth inhibition of the cells was observed (Figure S1A and S1B). To verify the effectiveness of the inhibitor in suppressing the PI3K pathway, we examined whether the phosphorylation levels of AKT were reduced following GDC-0326 treatment. As expected, we could observe that the expression level of p-AKT representing the activation of PI3K/AKT pathway was decreased significantly in the presence of GDC-0326, even in the lowest concentration of GDC-0326 (Figure S1C and S1D). As RIPK1 and RIPK3 are pivotal factors in necroptosis, we examined their expression in CRC cells exposed to GDC0326. As shown in Figure 1A, GDC-0326 induced the protein expression of RIPK1 and RIPK3 in both LoVo and HT-29 cells. In addition, colony formation assay demonstrated that GDC-0326 caused a decreased in the clonogenic growth of CRC cells; thus, GDC-0326 attenuated cell proliferation (Figure 1B and C). Additionally, immunofluorescence studies revealed accumulation of RIPK1 and RIPK3 after GDC-0326 treatment of the CRC cells (Figure 1D). These results indicate that the effects of GDC-0326 on the growth and viability of CRC cells involved RIPK1 and RIPK3.

To understand the clinical significance of RIPK1/3, data on their expression in CRC patients, as well as data on the prognosis of CRC patients, were extracted from the TCGA database. The data showed that the mRNA expression of RIPK1 and RIPK3 was lower in CRC tumor tissues than in adjacent normal tissues (Figure 2A and B). The median overall survival (OS) was much longer in the highRIPK3-expression group than in the low-RIPK3expression group. Although the difference in OS was not significant based on RIPK1 expression, patients with higher RIPK1 expression tended to have better OS ( $\mathrm{P}=$ 0.068) (Figure 2C and D).

\section{Reversal of GDC-0326-Induced Decrease in CRC Cell Viability by RIPKI/3}

\section{Inhibitors}

RIPK1 and RIPK3 expression was blocked by treatment of CRC cells with the specific inhibitors Nec-1 and GSK872, which directly bind to the active domains of RIPK1 and RIPK3, respectively. Nec-1 and GSK-872 blocked the upregulation of RIPK1 and RIPK3 protein expression, respectively, induced by GDC-0326 (Figure 3A and B). Further, the CCK-8 assay showed that co-culture with either Nec-1 or GSK-872 could partially reverse the death of CRC cells caused by GDC-0326 after $48 \mathrm{~h}$ of incubation (Figure 3C and D). This finding confirms that GDC-0326-induced CRC cell necroptosis was dependent on RIPK1 and RIPK3.

\section{Promotive Effect of GDC-0326 on the in vitro Effect of 5-Fu in CRC Cells}

CCK8 assay was used to determine that the $\mathrm{IC}_{50}$ value of 5-Fu for LoVo cells was 13.2 $\mu \mathrm{M}$ and that for HT-29 cells was $10.1 \mu \mathrm{M}$ (Figure 4A and B). In CRC cells that were treated with $5-\mathrm{Fu}$, the addition of GDC-0326 significantly reduced the cell viability (Figure $4 \mathrm{C}$ and $\mathrm{D}$ ). In addition, we analyzed the dose-dependent effect of the combination of 5-Fu and GDC-0326 in CRC cell lines and calculated the $\mathrm{CI}$. A CI value of less than 1 indicated that $5-\mathrm{Fu}$ and GDC-0326 had a synergistic effect on both LoVo and HT29 cells (Figure 4E and F).

\section{GDC-0326-Induced Increase in the in vivo Therapeutic Efficacy of 5-Fu}

To examine whether GDC-0326 and 5-Fu had a synergistic effect under in vivo conditions, a subcutaneous xenograft model with LoVo cells was established. Consecutive measurements of tumor volume demonstrated that the combination of GDC-0326 and 5-Fu had a more pronounced effect on slowing the tumor growth rate than $5-\mathrm{Fu}$ alone (Figure 5A). Additionally, a more obvious decrease in tumor weight was observed after the combination treatment (Figure 5B and $\mathrm{C}$ ), which had no impact on whole body weight.

Western blotting analysis revealed that combined treatment with 5-Fu and GDC-0326 significantly upregulated RIPK1 and RIPK3 levels compared to treatment with the 
A

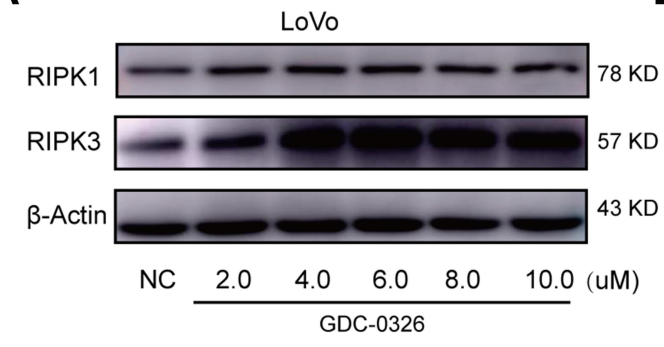

HT-29

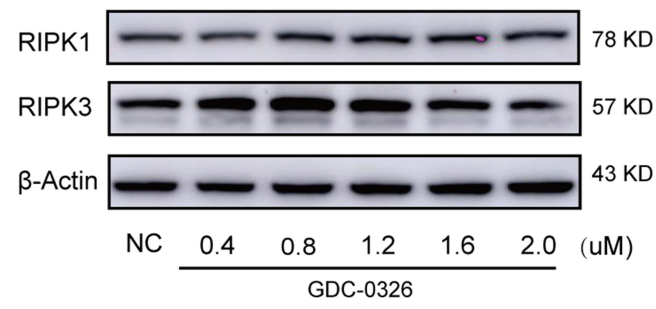

D
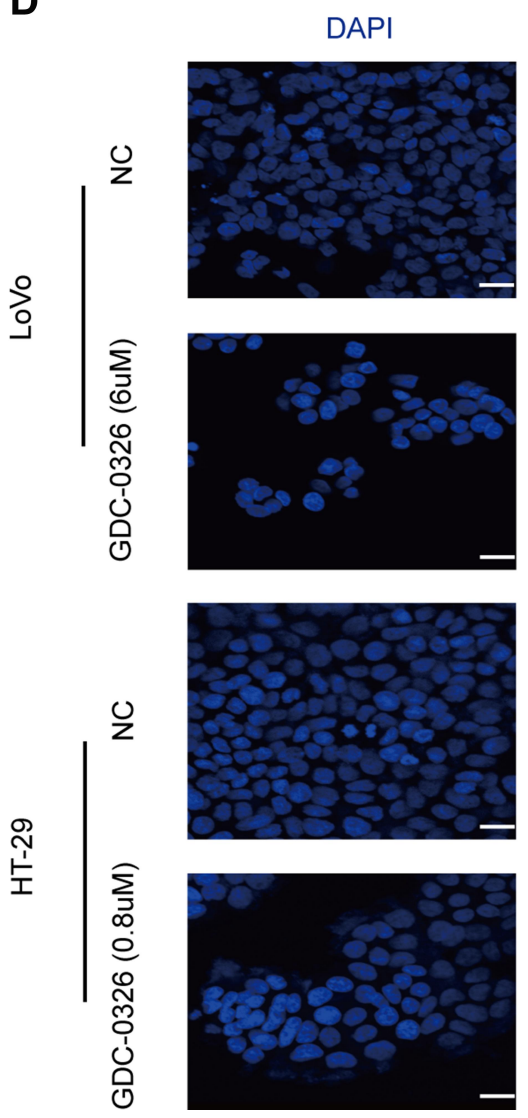

B

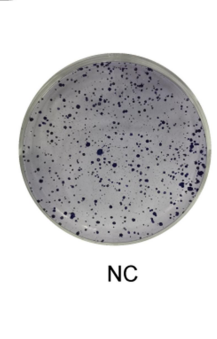

C

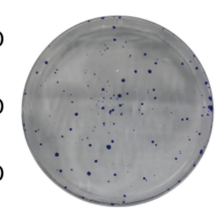

NC
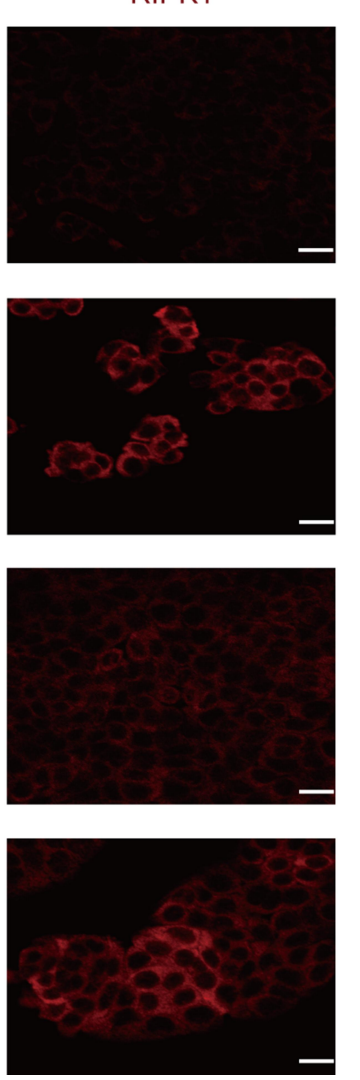

LoVo

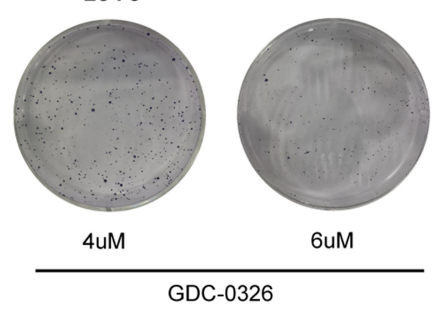

HT-29
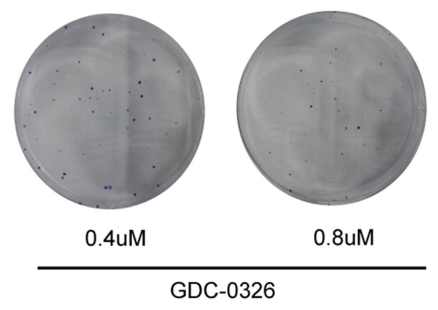

LoVo

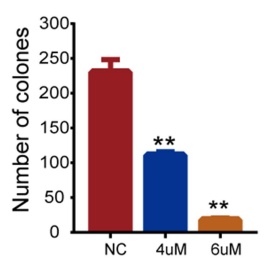

HT-29

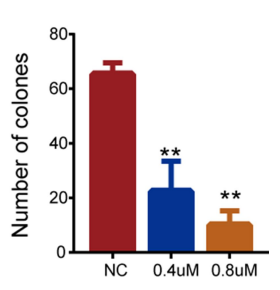

Figure I GDC-0326 induced inhibition of CRC cell proliferation through upregulation of RIPKI/3. (A) Protein levels of RIPKI and RIPK3 were detected by Western blotting after incubation with GDC-0326. (B and C) Colony formation of LoVo and HT-29 cells was observed, and the number of colonies formed was counted after 2 weeks of treatment with GDC-0326 at various concentrations. DMSO was added as a control. (D) Immunofluorescence images of RIPKI and RIPK 3 in CRC cells were captured after the addition of GDC-0326 for $48 \mathrm{~h}$. Scale bar $=20 \mu \mathrm{m}$. (**P $<0.01$ ).

vehicle only or treatment with either 5-Fu or GDC-0326 (Figure 5D). Additionally, histological analysis with H\&E and TUNEL staining showed that there were a higher number of necrotic cells in the tumors in the combination treatment group than in the only 5-Fu or only GDC-0326 treatment group (Figure 5E and F). 

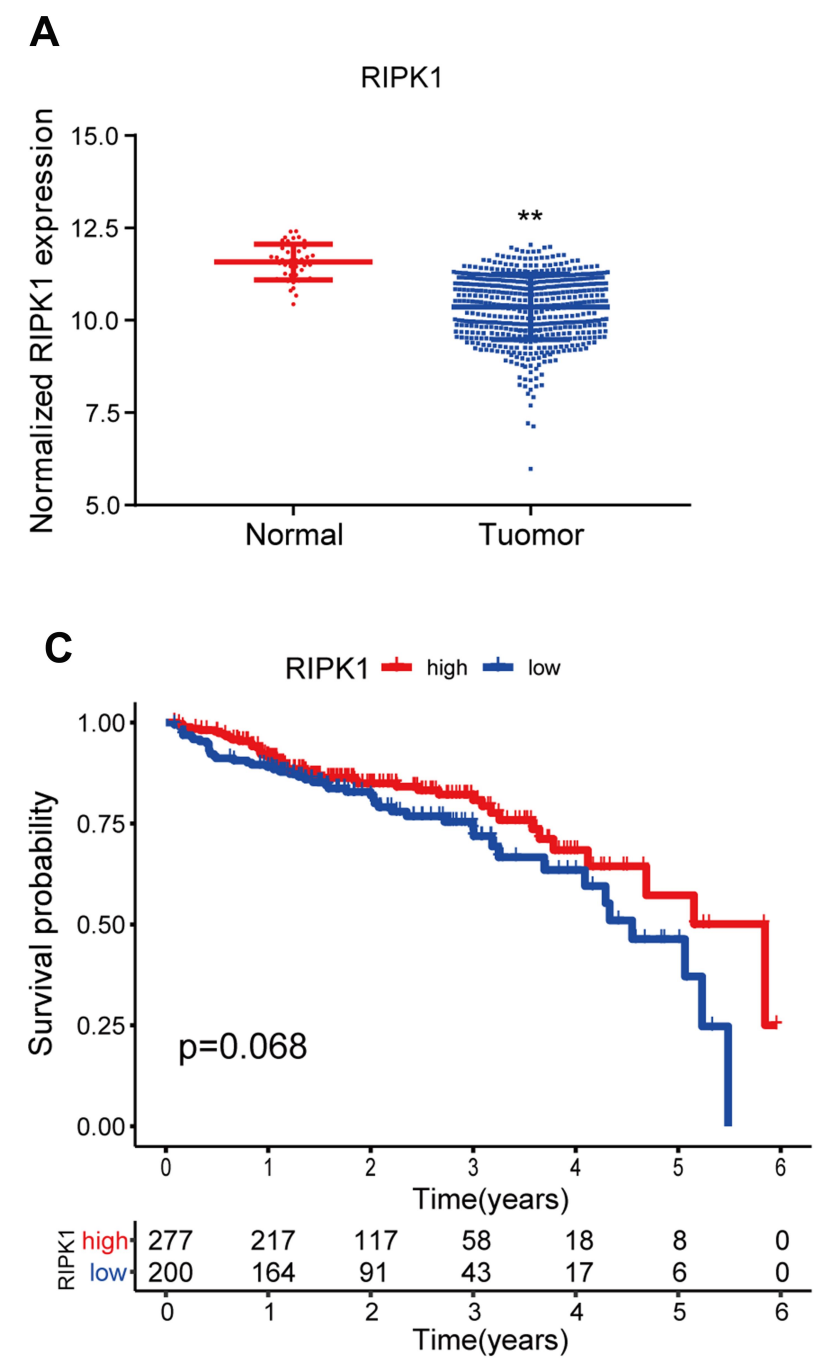

B
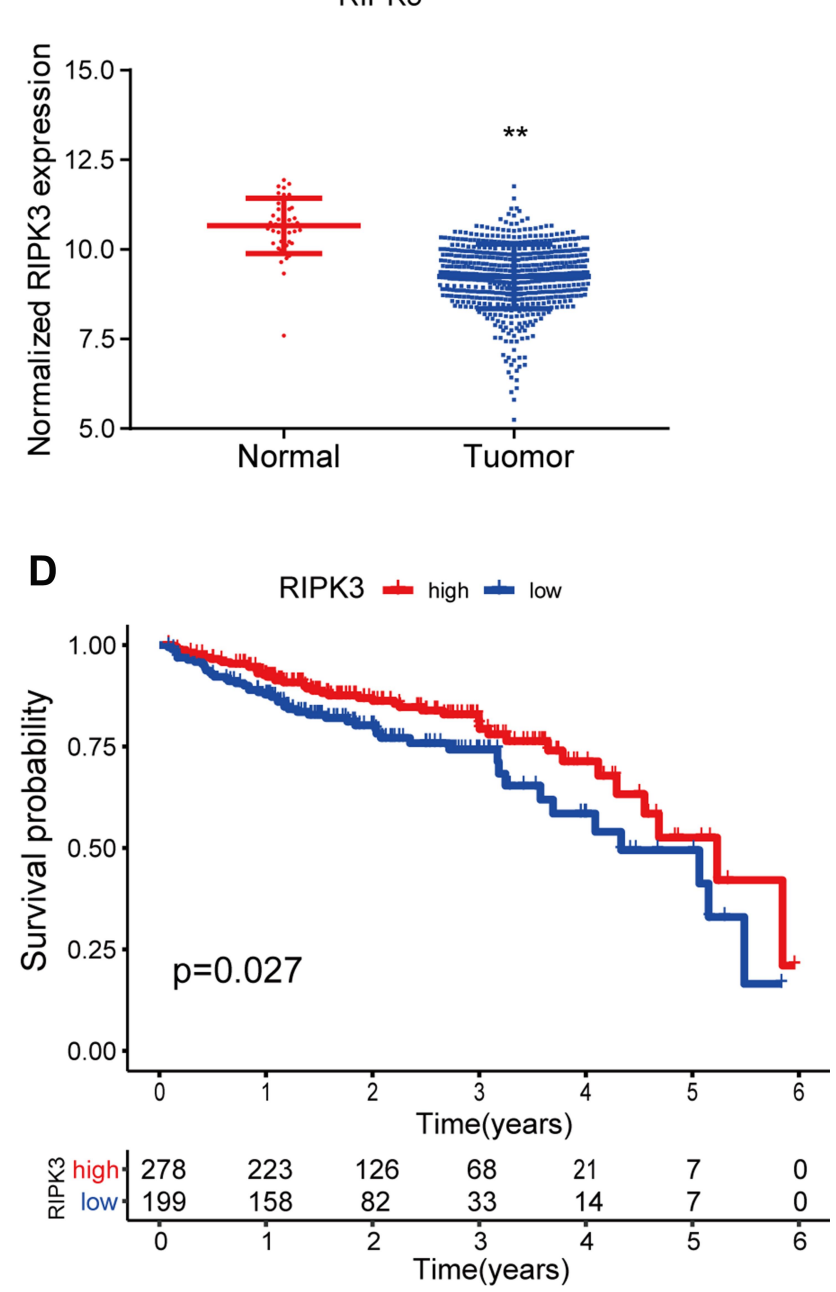

Figure 2 Clinical significance of RIPKI/3 expression in CRC based on the data extracted from TCGA. (A and B) Expression of RIPKI/3 was compared between tumor tissues and normal tissues in CRC. (C and D) Expression of RIPKI/3 was correlated with the prognosis of CRC patients. (**P $<0.0 \mathrm{I})$.

\section{Safety of GDC-0326 and 5-Fu Treatment Under in vivo Conditions}

Blood samples were drawn from the xenograft model mice after the completion of the other laboratory tests. The results revealed that WBC, RBC, and PLT counts, as well as in the hemoglobin level, between the three treatment groups were within a normal range (Figure 6A). Additionally, there were no significant differences in the biochemical indexes ALT, AST, TP, CR, and BUN between the groups (Figure 6B and C). Finally, histopathological analysis with $\mathrm{H} \& \mathrm{E}$ staining showed that GDC-0326 did not have a toxic effect on organs such as the heart, liver, spleen, lung, kidney, and intestine (Figure 6D).

\section{Discussion}

The present study examines whether the addition of GDC0326 as an adjuvant in 5-Fu treatment of CRC can improve the antitumor efficacy of 5-Fu and what the underlying mechanisms could be. The findings showed that administration of GDC-0326 enhanced the antitumor effect of 5-Fu on colorectal cancer cells by upregulating the levels of RIPK1 and RIPK3. This finding was confirmed by inhibition of either RIPK1 or RIPK3 with their specific corresponding inhibitors, which reversed GDC-0326-induced necroptosis of CRC cells.

Drug combination therapy has been widely used in malignant tumors, with the aim of achieving better efficacy and lower toxicity. ${ }^{27-30}$ For instance, shikonin, an 
A

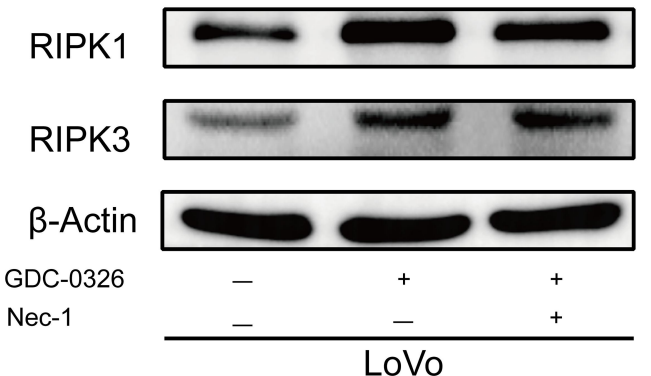

B
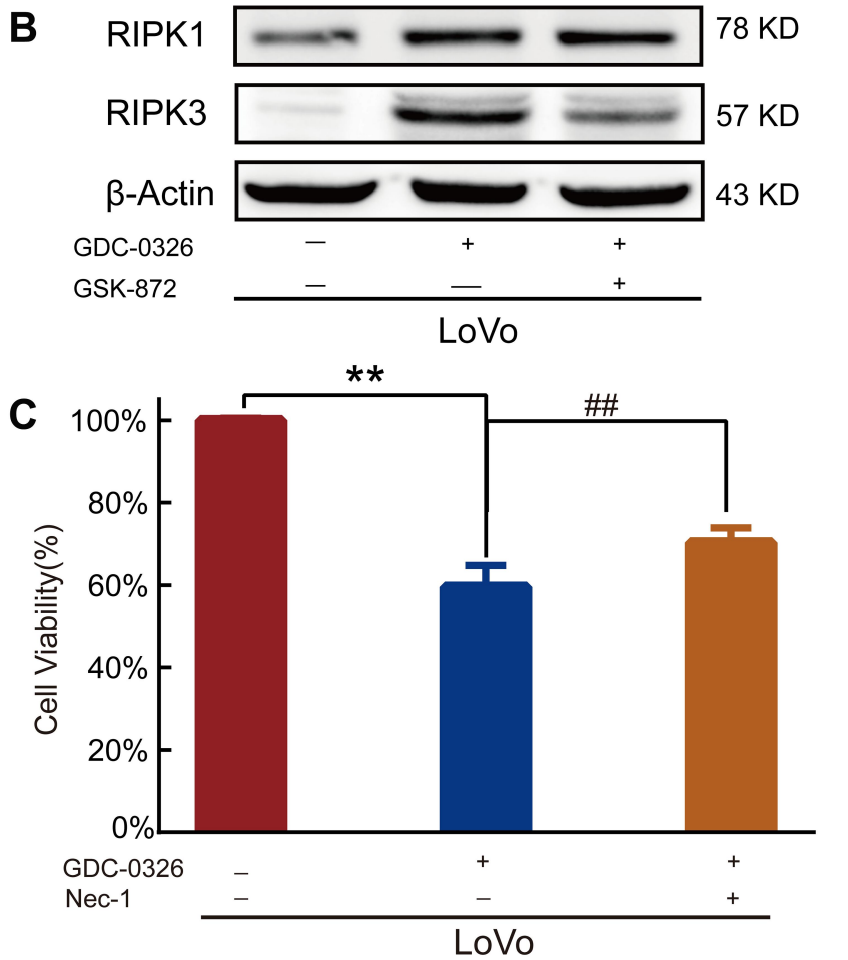

D

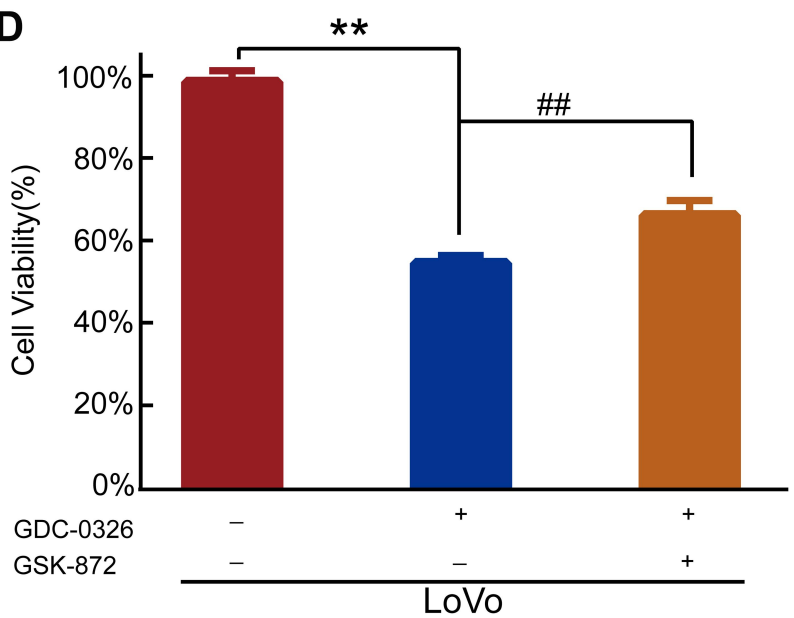

$78 \mathrm{KD}$

$57 \mathrm{KD}$

$43 \mathrm{KD}$

$78 \mathrm{KD}$

$57 \mathrm{KD}$

$43 \mathrm{KD}$

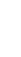

(1)
GDC-0326

$\mathrm{Nec}-1$

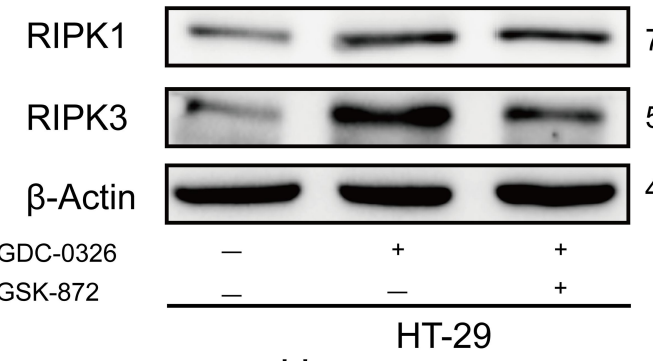

$78 \mathrm{KD}$

$57 \mathrm{KD}$
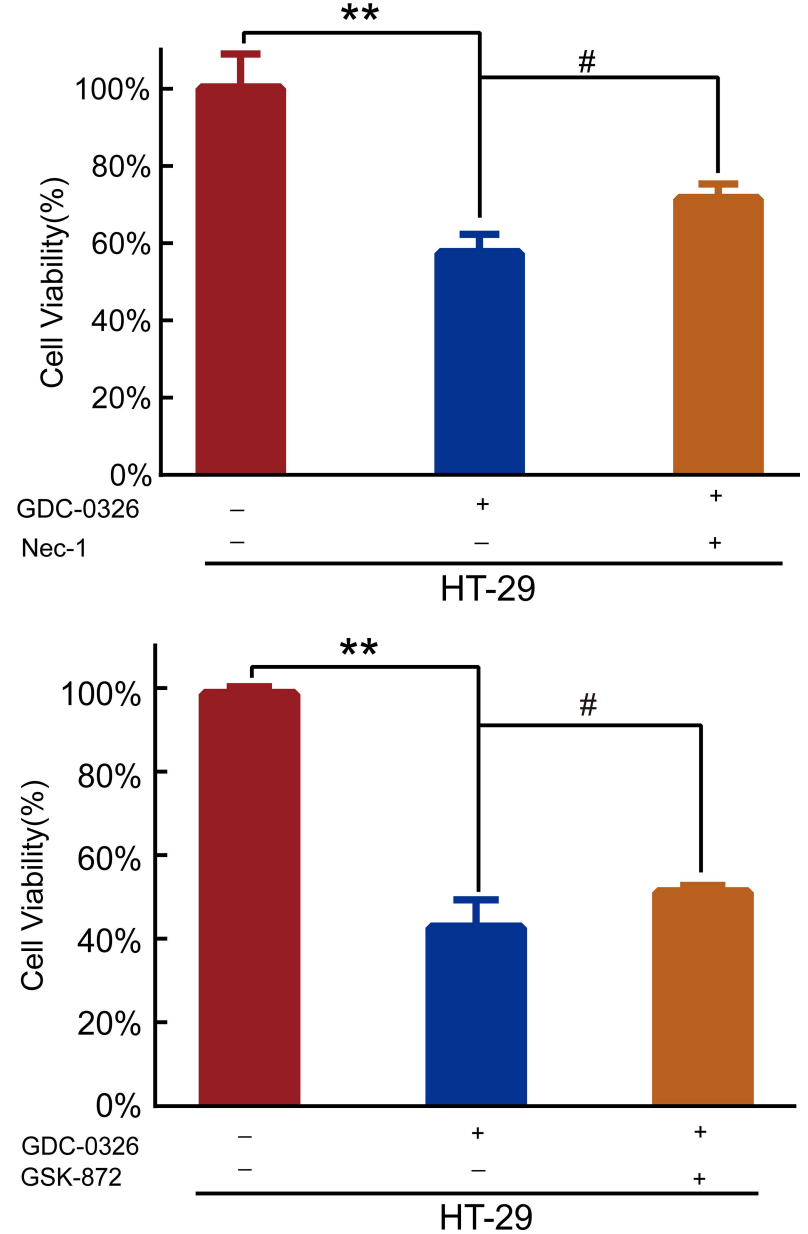

Figure 3 Effect of RIPKI/3 inhibitors on reversing the GDC-0326-induced decrease in CRC cell viability. (A and B) Western blot analysis revealed that Nec-I inhibited GDC-0326-induced upregulation of RIPKI, and GSK-872 mitigated the increase in RIPK3 expression induced by GDC-0326 in both LoVo and HT-29 cells. (C and D) CCK-8 assay showed that Nec-I and GSK-872 inhibited the death of CRC cells induced by GDC-0326. (**P $<0.01$, \#P $<0.05$, \#\#P $<0.01$ ).

extracted natural compound, has been found to enhance the anti-tumor effect of 5 -Fu under both in vitro and in vivo conditions. ${ }^{19}$ Based on these previous findings, in this study, we speculated that GDC-0326 could increase therapeutic efficacy of 5-Fu through activating necroptosis in CRC. Our findings revealed that GDC-0326 could 
A

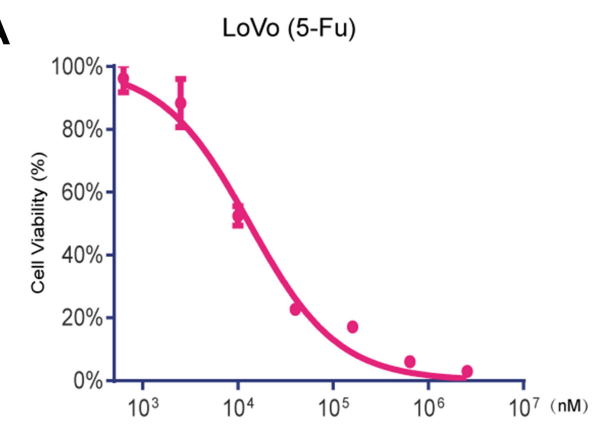

C

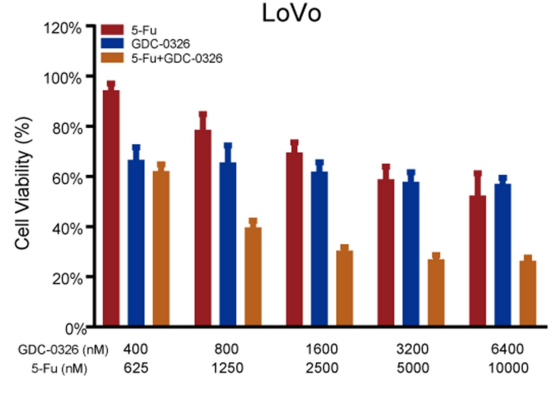

E

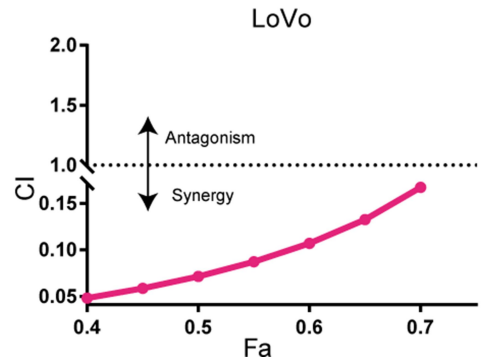

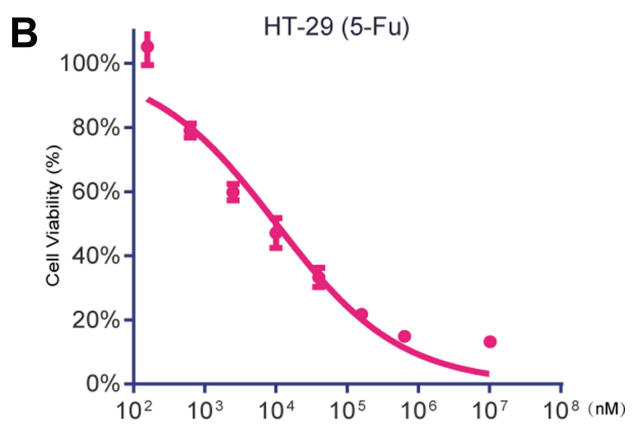

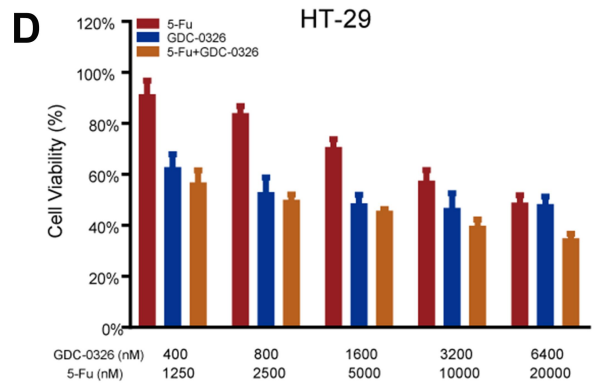

$\mathbf{F}$

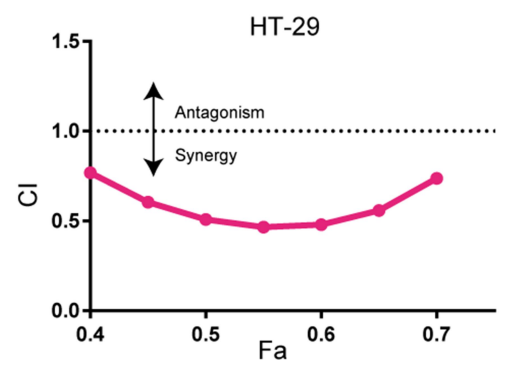

Figure 4 Promotive effect of GDC-0326 on improving the effect of 5-Fu in CRC cells. (A and B) The viability of LoVo and HT-29 cells was determined after incubation with 5-Fu for 48 h. (C and D) Cell viability was further assessed after treatment with various concentrations of GDC-0326 combined with 5-Fu. (E and F) The Cl plots of GDC0326 plus $5-\mathrm{Fu}$ in LoVo and HT-29 cells were delineated. $\mathrm{Cl}$, combination index $(\mathrm{Cl})$.

induce necroptosis by promoting the expression of RIPK1 and RIPK3 in human CRC cells. Accordingly, previous research has shown that RIPK3 deficiency facilitated immune escape and progression of hepatocellular carcinoma. ${ }^{31}$ Additionally, in the present study, survival analysis of data from the TGCA database demonstrated that the level of RIPK1 and RIPK3 in primary tumors might be a potential predictor of prognosis. In the near future, we will investigate whether the expression of RIPK1 and RIPK3 in CRC can also be applied as a predictor of sensitivity to $5-\mathrm{Fu}$.

Most chemotherapy agents are cytotoxic and inevitably have adverse effects, and this limits their clinical use for the treatment of CRC. Therefore, in the present study, we investigated potential cytotoxic effects of GDC-0326. Fortunately, it did not exhibit any obvious hepatotoxicity or nephrotoxicity in mouse xenograft models of CRC. Additionally, bone marrow suppression is a common and undesirable side effect of many conventional chemotherapeutic agents. Encouragingly, no obvious myelosuppression was observed in the nude mice in this study. Thus, our findings confirm the clinical safety of GDC-0326.

Some limitations of our findings must be noted here. Although the findings confirm the enhanced necroptotic effect of GDC-0326 on CRC cells, the underlying mechanism could not be completely elucidated. Recent studies have proved the relationship between the PI3K/AKT/ mTOR signaling pathway and necroptosis, and the PI3K signaling pathway is known to play a major role in regulating the proliferation, cell cycle and metastasis of CRC cells. ${ }^{32}$ Additionally, other chemotherapeutic agents, such as FTY720, have been found to induce human glioblastoma cell necroptosis via inhibition of the PI3K/AKT pathway. ${ }^{33}$ Other mechanisms, such as upregulation of glycolipid transfer protein via RIPK3, have also been found to induce necroptosis. ${ }^{34}$ Therefore, in the future, it would be useful to investigate whether the necroptotic effect of GDC-0326 is mediated by PI3K/AKT/mTOR or 
A

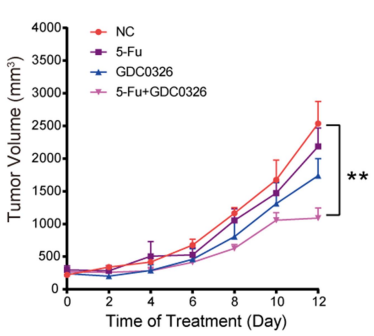

B

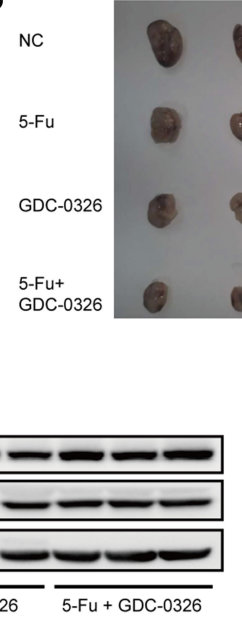

D

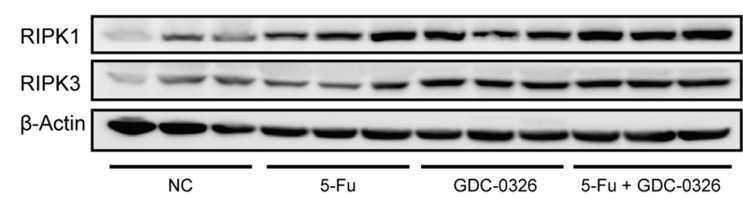

$\mathbf{F}$
5-Fu
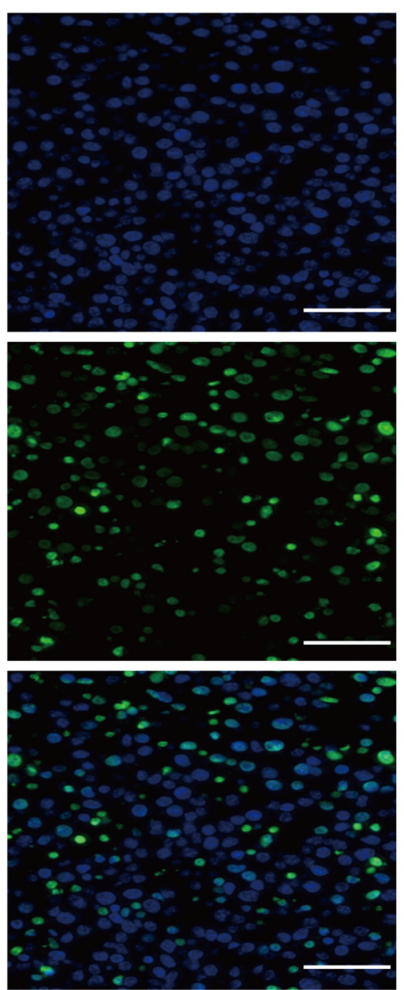

E

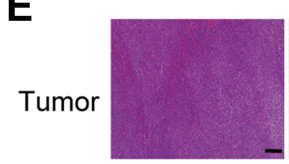

NC
C

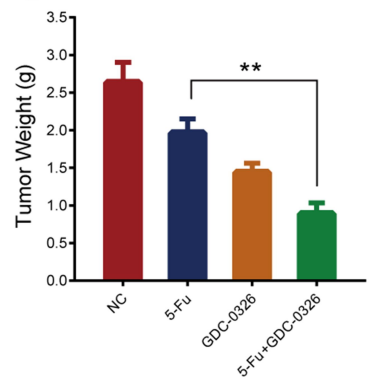

DAPI

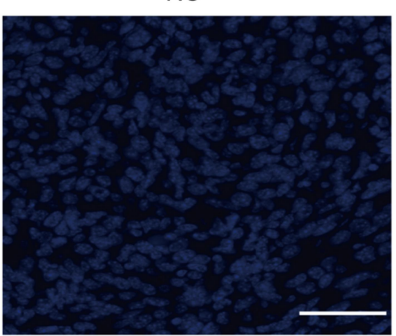

GDC-0326

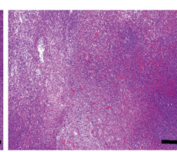

5-Fu

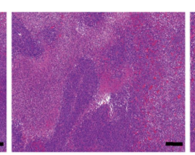

GDC-0326

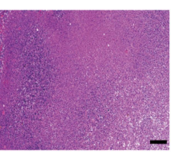

5-Fu + GDC-0326
5-Fu + GDC-0326
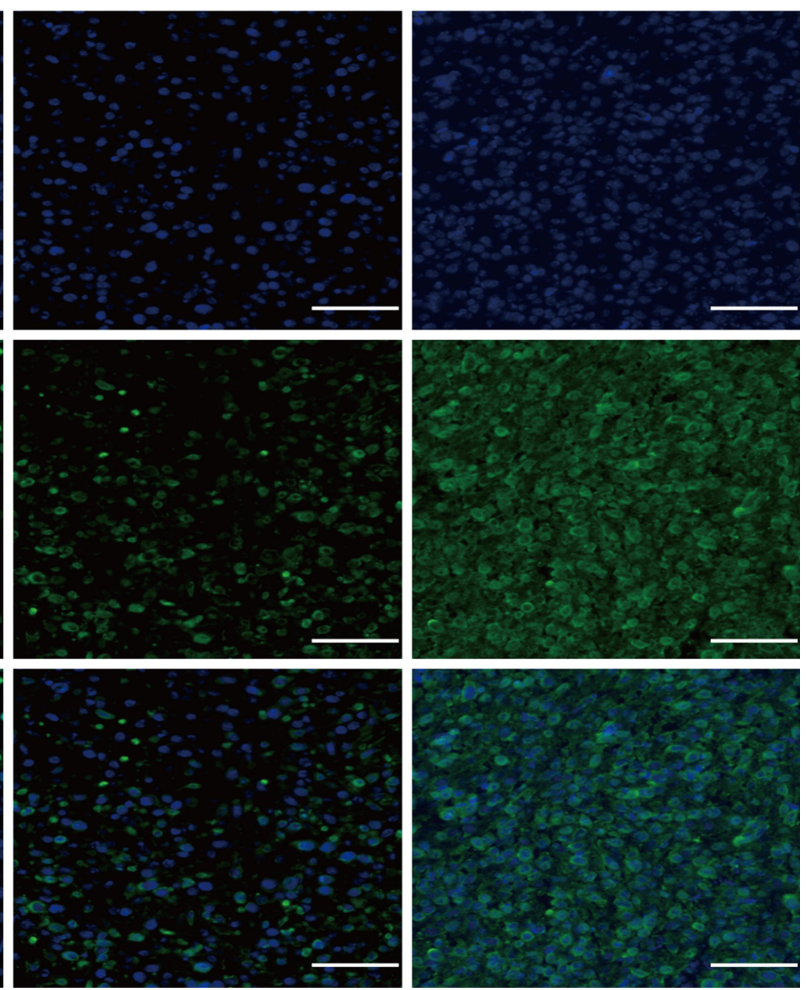

Figure 5 GDC-0326 enhanced the effects of 5-Fu on suppressing CRC tumor growth in vivo. (A) Growth curve of tumor volumes after injection of LoVo cells in xenograft nude mice from the DMSO, 5-Fu, GDC-0326, and combination treatment groups. (B and C) The xenografted tumors were surgically removed and photographed, as well as weighed $(n=5)$. (D) Protein levels of RIPKI and RIPK3 were detected by Western blotting analysis in each group. $\beta$-Actin was used as a loading control. (E) H\&E staining of tumor slices from different groups after various treatments (scale bar $=200 \mu \mathrm{m})$. $(\mathbf{F})$ TUNEL immunofluorescence staining of tumor slices from the groups $($ scale bar $=100$ $\mu \mathrm{m}) .(* * \mathrm{P}<0.01)$.

other signaling pathways. Another limitation of GDC0326 is that it is insoluble in water. Therefore, it is important to investigate methods to increase its solubility. Nanocrystallization is a promising strategy to increase the apparent solubility, dissolution rate, and oral bioavailability of hydrophobic drugs. ${ }^{35,36}$ A nanoscale drug delivery system might improve the oral bioavailability of GDC-0326, and thus, facilitate its antitumor and synergistic effect when used in combination with 5 -Fu. This is a line of investigation that needs to be pursued in the future for improving the clinical applicability of GDC0326 . 

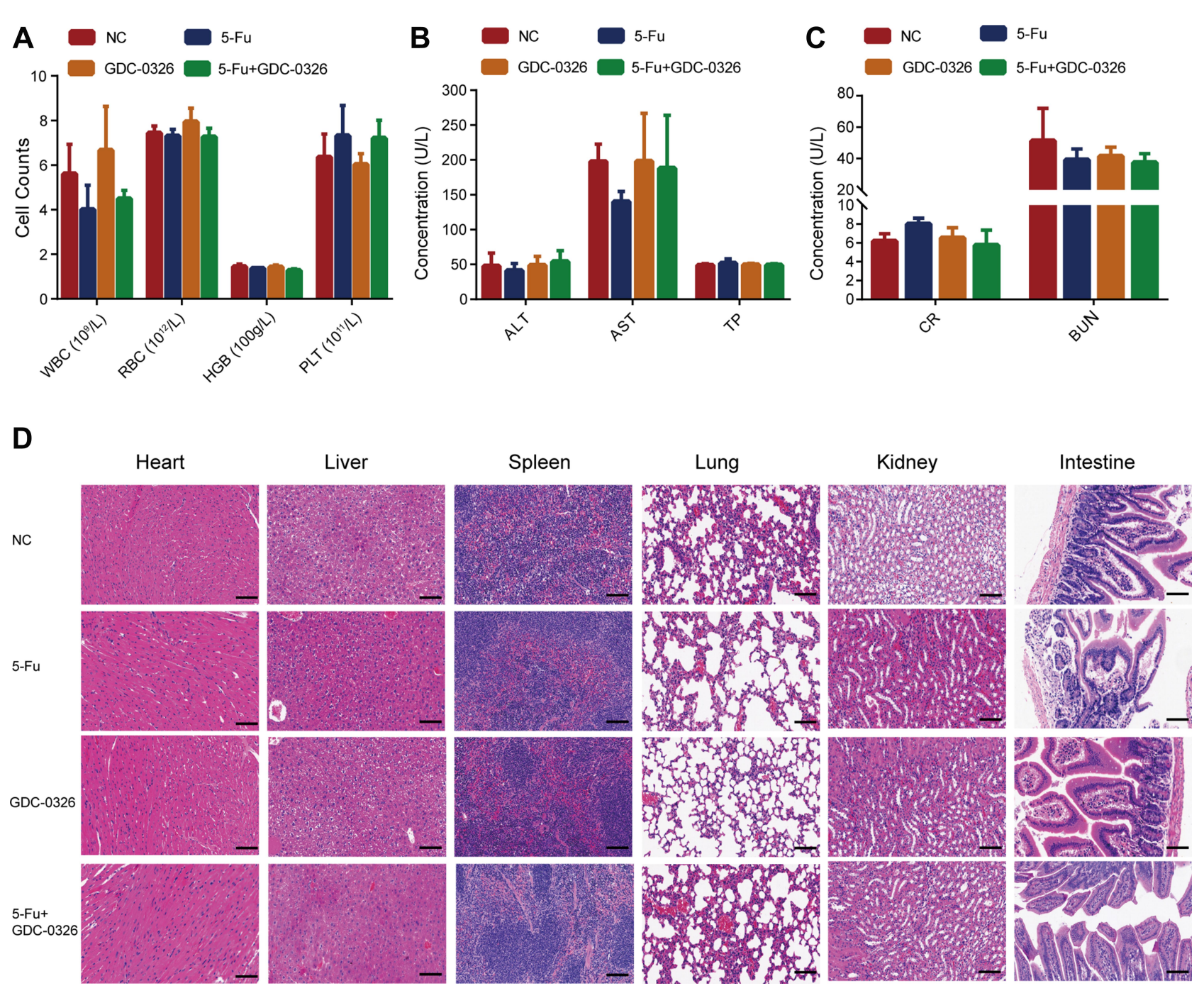

Figure 6 Safety of combination therapy with GDC-0326 and 5-Fu in tumor-bearing mice. (A-C) Blood routine analysis and biochemical tests were performed in the mice, including white blood cell (WBC), red blood cell (RBC), hemoglobin (HGB), platelet (PLT), alanine transaminase (ALT), aspartate transaminase (AST), total protein (TP), creatinine (CR), and blood urea nitrogen (BUN) tests. For quantitative data, the total number of mice used was 5. (D) H\&E staining images of tissues from tumor-bearing mice, including tissue from the heart, liver, spleen, lung, kidney, and intestine (scale bar $=100 \mu \mathrm{m}$ ).

\section{Conclusion}

Our data demonstrated that GDC-0326 induced necroptosis of $\mathrm{CRC}$ cells treated with 5 -Fu by enhancing the expression of RIPK1 and RIPK3. Additionally, the combination therapy of 5-Fu and GDC-0326 was found to be safe for use under in vivo conditions. Thus, this drug combination has great potential in the chemotherapeutic management of CRC.

\section{Abbreviations}

CRC, Colorectal cancer; 5-Fu, 5-fluorouracil; RIPK, Receptor interacting serine/threonine kinase; GLTP, glycolipid transfer protein; p-MLKL, phosphorylating mixedlineage kinase domain-like; CCK-8, Cell Counting Kit-8; OS, Overall survival. CI, combination index; H\&E, hematoxylin-eosin; TUNEL, TdT-mediated dUTP nick end labeling; HCC, hepatocellular carcinoma; MDSC, myeloid-derived suppressor cell; WBC, white blood cell; RBC, red blood cell; HGB, hemoglobin; PLT, platelet; ALT, alanine transaminase; AST, aspartate transaminase; $\mathrm{TP}$, total protein; $\mathrm{CR}$, creatinine; BUN, blood urea nitrogen.

\section{Funding}

This work was supported by the National Natural Science Foundation of China (grant nos. 82073229 and 81702308), Zhejiang Provincial Natural Science Foundation (grant nos. LY18H160009 and LY16H160031), and the National Natural Science Foundation of China (grant no. 81802348). 


\section{Disclosure}

The authors report no conflicts of interest in this work.

\section{References}

1. Wang JH, Lu TJ, Kung ML, et al. The long noncoding RNA LOC441461 (STX17-AS1) modulates colorectal cancer cell growth and motility. Cancers. 2020;12(11):3171. doi:10.3390/ cancers 12113171

2. Bray F, Ferlay J, Soerjomataram I, et al. Global cancer statistics 2018: GLOBOCAN estimates of incidence and mortality worldwide for 36 cancers in 185 countries. CA Cancer J Clin. 2018;68 (6):394-424. doi:10.3322/caac.21492

3. Dittrich L, Biebl M, Schmuck R, et al. Initial experience with the safe implementation of Transanal Total Mesorectal Excision (TaTME) as a standardized procedure for low rectal cancer. J Clin Med. 2020;10 (1):72. doi:10.3390/jcm10010072

4. Fletcher JI, Haber M, Henderson MJ, Norris MD. ABC transporters in cancer: more than just drug efflux pumps. Nat Rev Cancer. 2010;10(2):147-156. doi:10.1038/nrc2789

5. Wosikowski K, Schuurhuis D, Kops GJ, Saceda M, Bates SE. Altered gene expression in drug-resistant human breast cancer cells. Clin Cancer Res. 1997;3(12 Pt 1):2405-2414.

6. Zhang YH, Luo DD, Wan SB, Qu XJ. S1PR2 inhibitors potently reverse 5-FU resistance by downregulating DPD expression in colorectal cancer. Pharmacol Res. 2020;155:104717. doi:10.1016/j. phrs.2020.104717

7. Wang W, Guo W, Li L, et al. Andrographolide reversed 5-FU resistance in human colorectal cancer by elevating BAX expression. Biochem Pharmacol. 2016;121:8-17. doi:10.1016/j.bcp.2016.09.024

8. Țigu AB, Toma VA, Moț AC, et al. The synergistic antitumor effect of 5-fluorouracil combined with allicin against lung and colorectal carcinoma cells. Molecules. 2020;25(8):1947. doi:10.3390/ molecules 25081947

9. Feng Y, Gao Y, Wang D, et al. Autophagy inhibitor (LY294002) and 5-fluorouracil (5-FU) combination-based nanoliposome for enhanced efficacy against esophageal squamous cell carcinoma. Nanoscale Res Lett. 2018;13(1):325. doi:10.1186/s11671-018-2716-X

10. Liu F, Ai F-Y, Zhang D-C, et al. LncRNA NEAT1 knockdown attenuates autophagy to elevate 5-FU sensitivity in colorectal cancer via targeting miR-34a. Cancer Med. 2020;9(3):1079-1091. doi:10.1002/cam4.2746

11. Gao J, Qiu X, Xi G, et al. Downregulation of GSDMD attenuates tumor proliferation via the intrinsic mitochondrial apoptotic pathway and inhibition of EGFR/Akt signaling and predicts a good prognosis in nonsmall cell lung cancer. Oncol Rep. 2018;40(4):1971-1984. doi:10.3892/or.2018.6634

12. Zhang Z, Zhang Y, Xia S, et al. Gasdermin E suppresses tumour growth by activating anti-tumour immunity. Nature. 2020;579 (7799):415-420. doi:10.1038/s41586-020-2071-9

13. Zhu Y, Hu H, Yuan Z, et al. LncRNA NEAT1 remodels chromatin to promote the $5-\mathrm{Fu}$ resistance by maintaining colorectal cancer stemness. Cell Death Dis. 2020;11(11):962. doi:10.1038/s41419020-03164-8

14. Kroemer G, Galluzzi L, Vandenabeele P, et al. Classification of cell death: recommendations of the nomenclature committee on cell death 2009. Cell Death Differ. 2009;16(1):3-11. doi:10.1038/cdd.2008.150

15. Lu X, Fu H, Chen R, et al. Phosphoinositide specific phospholipase $\mathrm{C} \gamma 1$ inhibition-driven autophagy caused cell death in human lung adenocarcinoma A549 cells in vivo and in vitro. Int $J$ Biol Sci. 2020;16(8):1427-1440. doi:10.7150/ijbs.42962
16. Christofferson DE, Yuan J. Necroptosis as an alternative form of programmed cell death. Curr Opin Cell Biol. 2010;22(2):263-268. doi:10.1016/j.ceb.2009.12.003

17. Zheng W, Zhou CY, Zhu XQ, et al. Oridonin enhances the cytotoxicity of 5-FU in renal carcinoma cells by inducting necroptotic death. Biomed Pharmacother. 2018;106:175-182. doi:10.1016/j. biopha.2018.06.111

18. Linkermann A, Green DR. Necroptosis. $N$ Engl J Med. 2014;370 (5):455-465. doi:10.1056/NEJMra1310050

19. Chen C, Xiao W, Huang L, et al. Shikonin induces apoptosis and necroptosis in pancreatic cancer via regulating the expression of RIP1/RIP3 and synergizes the activity of gemcitabine. Am J Transl Res. 2017;9(12):5507-5517.

20. Vivanco I, Sawyers CL. The phosphatidylinositol 3-kinase AKT pathway in human cancer. Nat Rev Cancer. 2002;2(7):489-501. doi:10.1038/nrc839

21. Fritsch C, Huang A, Chatenay-Rivauday C, et al. Characterization of the novel and specific PI3Kalpha inhibitor NVP-BYL719 and development of the patient stratification strategy for clinical trials. Mol Cancer Ther. 2014;13(5):1117-1129. doi:10.1158/1535-7163.MCT-13-0865

22. Furet P, Guagnano V, Fairhurst RA, et al. Discovery of NVP-BYL719 a potent and selective phosphatidylinositol-3 kinase alpha inhibitor selected for clinical evaluation. Bioorg Med Chem Lett. 2013;23 (13):3741-3748. doi:10.1016/j.bmcl.2013.05.007

23. McNamara CR, Ahuja R, Osafo-Addo AD, et al. Akt regulates TNFalpha synthesis downstream of RIP1 kinase activation during necroptosis. PLoS One. 2013;8(3):e56576. doi:10.1371/journal. pone. 0056576

24. Soler A, Figueiredo AM, Castel P, et al. Therapeutic benefit of selective inhibition of p110alpha PI3-kinase in pancreatic neuroendocrine tumors. Clin Cancer Res. 2016;22(23):5805-5817. doi:10.1158/ 1078-0432.CCR-15-3051

25. Heffron TP, Heald RA, Ndubaku C, et al. The rational design of selective benzoxazepin inhibitors of the alpha-isoform of phosphoinositide 3-kinase culminating in the identification of (S)-2-((2-(1-Isopropyl-1H-1,2,4-triazol-5-yl)-5,6-dihydrobenzo[f]imidazo[1,2-d][1,4]oxazepin-9-yl)oxy)propanamide (GDC-0326). $J$ Med Chem. 2016;59(3):985-1002. doi:10.1021/acs.jmedchem.5b01483

26. Chou TC. Drug combination studies and their synergy quantification using the Chou-Talalay method. Cancer Res. 2010;70(2):440-446. doi:10.1158/0008-5472.Can-09-1947

27. Ashton JC. Drug combination studies and their synergy quantification using the Chou-Talalay method-letter. Cancer Res. 2015;75 (11):2400. doi:10.1158/0008-5472.CAN-14-3763

28. Sinzger M, Vanhoefer J, Loos C, Hasenauer J. Comparison of null models for combination drug therapy reveals hand model as biochemically most plausible. Sci Rep. 2019;9(1):3002. doi:10.1038/ s41598-019-38907-x

29. Scheiblecker L, Kollmann K, Sexl V. CDK4/6 and MAPK-crosstalk as opportunity for cancer treatment. Pharmaceuticals. 2020;13 (12):418. doi:10.3390/ph13120418

30. Ji M, Li Z, Lin Z, Chen L. Antitumor activity of the novel HDAC inhibitor CUDC-101 combined with gemcitabine in pancreatic cancer. Am J Cancer Res. 2018;8(12):2402-2418.

31. Li YM, Liu ZY, Wang JC, et al. Receptor-interacting protein kinase 3 deficiency recruits myeloid-derived suppressor cells to hepatocellular carcinoma through the chemokine (C-X-C motif) ligand 1-chemokine (C-X-C motif) receptor 2 axis. Hepatology. 2019;70(5):1564-1581. doi:10.1002/hep.30676

32. Zhang J, Roberts TM, Shivdasani RA. Targeting PI3K signaling as a therapeutic approach for colorectal cancer. Gastroenterology. 2011;141(1):50-61. doi:10.1053/j.gastro.2011.05.010 
33. Zhang L, Wang H, Ding K, Xu J. FTY720 induces autophagy-related apoptosis and necroptosis in human glioblastoma cells. Toxicol Lett. 2015;236(1):43-59. doi:10.1016/j.toxlet.2015.04.015

34. Mishra SK, Stephenson DJ, Chalfant CE, Brown RE. Upregulation of human glycolipid transfer protein (GLTP) induces necroptosis in colon carcinoma cells. Biochim Biophys Acta Mol Cell Biol Lipids. 2019;1864(2):158-167. doi:10.1016/j.bbalip.2018.11.002
35. Xin Y, Huang Q, Tang JQ, et al. Nanoscale drug delivery for targeted chemotherapy. Cancer Lett. 2016;379(1):24-31. doi:10.1016/j. canlet.2016.05.023

36. Ding Z, Wang L, Xing Y, et al. Enhanced oral bioavailability of celecoxib nanocrystalline solid dispersion based on wet media milling technique: formulation, optimization and in vitro/in vivo evaluation. Pharmaceutics. 2019;11(7):328. doi:10.3390/pharmaceutics11070328

\section{Publish your work in this journal}

OncoTargets and Therapy is an international, peer-reviewed, open access journal focusing on the pathological basis of all cancers, potential targets for therapy and treatment protocols employed to improve the management of cancer patients. The journal also focuses on the impact of management programs and new therapeutic

Submit your manuscript here: https://www.dovepress.com/oncotargets-and-therapy-journal agents and protocols on patient perspectives such as quality of life, adherence and satisfaction. The manuscript management system is completely online and includes a very quick and fair peer-review system, which is all easy to use. Visit http://www.dovepress.com/ testimonials.php to read real quotes from published authors. 